variables, or use longer names, you can alter the values assigned to VAR\% and CHAR\% respectively on line 20 of FILER.

\title{
19 How can I use a graphics dump?
}

A graphics dump routine is not included with STATCALC because it would have to be tailored to your specific printer. Many graphics dumps load at \&A00 and this assumption is made in UNIVAR and BIVAR. You can easily find out where your dump loads by typing $*$ OPT 1,2 and then $*$ LOAD your dump routine. The first number that appears on the screen after the name of the routine is the required load address. If your graphics dump does not load at \&A00 then you will need to alter line 270 in UNIVAR and line 1143 in BIVAR.

Before you use UNIVAR or BIVAR (either via the MENU program or by explicitly CHAINing them) you must first *LOAD your graphics routine. Whilst you are running UNIVAR or BIVAR and the screen is displaying graphics, press the key marked @ in order to obtain a copy on your printer. On completion, you will be returned to the program. It is your responsibility to ensure that the printer is switched on and connected to the microcomputer.

Not all dump routines will work with UNIVAR or BIVAR because they use load addresses which are overwritten by the DFS when the programs retrieve data files. 